\title{
ParS-independent recruitment of the bacterial chromosome-partitioning protein ParB
}

\author{
Miloš Tišma ${ }^{1}$, Maria Panoukidou ${ }^{3}$, Hammam Antar ${ }^{2}$, Young-Min Soh², Roman Barth ${ }^{1}$, Biswajit \\ Pradhan $^{1}$, Jaco van der Torre ${ }^{1}$, Davide Michieletto $^{3,4},{\text { Stephan } \text { Gruber }^{2}, \text { Cees Dekker }}^{1^{*}}$
}

\author{
${ }^{1}$ Department of Bionanoscience, Kavli Institute of Nanoscience Delft, Delft University of \\ Technology; Delft, Netherlands. \\ ${ }^{2}$ Department of Fundamental Microbiology (DMF), Faculty of Biology and Medicine (FBM), \\ University of Lausanne (UNIL); Lausanne, Switzerland. \\ ${ }^{3}$ School of Physics and Astronomy, University of Edinburgh, Edinburgh, UK. \\ ${ }^{4}$ MRC Human Genetics Unit, Institute of Genetics and Cancer, University of Edinburgh, \\ Edinburgh, UK. \\ *Corresponding author: C.Dekker@tudelft.nl
}

\begin{abstract}
The ParABS system plays an essential role in prokaryotic chromosome segregation. After loading at the parS site on the genome, ParB proteins rapidly redistribute to distances of $\sim 15 \mathrm{~kb}$ away from the loading site. It has remained puzzling how this large-distance spreading can occur along DNA that is loaded with hundreds of proteins. Using single-molecule in vitro visualization, we here show that, unexpectedly, ParB can load onto DNA independently and distantly of parS, whereby loaded ParB molecules are themselves able to recruit additional ParB proteins from bulk. Strikingly, this recruitment can occur in-cis but also in-trans whereby, at low tensions within the DNA, newly recruited ParB can bypass roadblocks as it gets loaded to spatially proximal but genomically distant DNA regions. The data are supported by Molecular Dynamics simulations which also show that cooperative ParB-ParB recruitment enhances spreading. ParS-independent recruitment explains how ParB can cover substantial genomic distance during chromosome segregation which is vital for the bacterial cell cycle.
\end{abstract}


Accurate chromosome segregation is crucial for a stable transmission of genetic material through each cell cycle. To actively segregate origins of replication, most prokaryotes rely on the ParABS system ( 1 , 2), which consists of a parS binding sequence, the ATPase partition protein A (ParA), and the CTPase partition protein B (ParB) (3-6). ParB dimers bind to the parS sequence, located near the origin of replication, spread laterally and ultimately form ParB-DNA partition complexes that are imperative for DNA segregation (7-13). A ParA gradient along the cell axis subsequently segregates these complexes to effectively administer the nascent genomes to the daughter cells $(14,15)$. Proper partitioning of the ParB complexes is vital for bacterial cell survival and has been studied intensively in recent years (16), especially since it was recently discovered that ParB proteins from several model organisms utilize CTP to load onto a parS sequence $(4,5,17)$.

A necessary feature for correct chromosome partitioning comes from a particular ability of ParB proteins to clamp the parS-DNA as a dimer $(4,5)$ and to subsequently slide along the DNA by diffusion, effectively freeing the parS site for new ParB proteins to load. Indeed, ParB has been found to laterally spread over large genomic regions surrounding the parS sites in vivo (10-15 kb; (18-22)), which was reported to be essential for partition complex formation $(9,10,16,18,19,23-25)$. However, in vitro studies showed that single DNA-bound proteins block the diffusion of ParB along DNA very efficiently $(4,7,17)$, raising the question how spreading can occur in a dense cellular environment, where, with $\sim 1$ gene and $\sim 20-50$ Nucleoid Associated Proteins (NAPs) per kb $(26,27)$, sliding ParB dimers will continuously run into 'roadblocks' that will stall their movement. Indeed, theoretical modelling of a ParB 'clamping and sliding' model indicated that such roadblocks dramatically limit the spreading distance on F-plasmids (28). Yet, in vivo ChIP-seq data do not show strong changes in the ParB occupancy in the vicinity of genes and operons $(18-22,29)$.

Here, we examine the mechanism of ParB spreading using a controlled DNA-stretching assay that allows in vitro visualization at the single-molecule level (30). First, we verified that CTP and the parS site are essential for the loading and diffusion of ParB on DNA. We tethered $42 \mathrm{~kb}$ long DNA molecules with a single $\operatorname{par} S$ site close to the middle $\left(\mathrm{DNA}_{\text {par } S}\right)$ to a streptavidin-functionalized surface via their 5'-biotin ends (Fig. 1A, S1A). Highly Inclined and Laminated Optical sheet microscopy was used to observe DNA and proteins labelled with different fluorescent dyes (see Supplementary info, Fig. S1B-F). ParB was found to exhibit two different types of behaviors, viz., transient non-diffusive binding at various locations (Fig.S2A-C), as well as binding at the parS site followed by diffusion (Fig. 1B and S2D-E). Transient binding was short ( 5-20 s), independent of the presence of CTP in the medium, and not correlated to the parS sequence (Fig 1C, S2B-C). In the presence of both the parS sequence and CTP, however, binding initiated predominantly at the location of the parS sequence (Fig. 1D and Fig. S2D-H). This binding persisted for an average time of $94 \pm 8$ seconds (average \pm SEM; Fig. 1E), during 
which time the ParB diffused away from the parS site. This residence time is in line with the previously determined CTP hydrolysis rate of 1 CTP per $100 \mathrm{~s}$ for $\operatorname{ParB}_{B S u}(4)$, where the hydrolysis of CTP would cause the ParB clamp to destabilize and open, allowing dissociation from the DNA $(4,5,19)$. Interestingly, we also observed a minor second population of molecules with roughly double the residence time of the first population $(188 \pm 18 \mathrm{~s}$ (average \pm SEM), Fig. 1E, S3). This strengthens previous indications that ParB dimers can prolong their residence time on DNA by exchanging CTP molecules after loading (19).

Strikingly, the data also revealed an unexpected behavior that was different from mere loading and diffusion of ParB dimers: a ParB dimer that was previously loaded onto the $\mathrm{DNA}_{\text {pars }}$, was observed to be able to load a new ParB dimer onto the DNA (Fig. 1F, G and S4A-D). This behavior was characterized by the recruitment of a second ParB dimer at the site of an existing one (as evidenced from an increase of the fluorescent intensity), a brief colocalization and conjunct diffusion of the two ParB dimers (for $8.2 \pm 3.5 \mathrm{~s}$, average $\pm \mathrm{SD}$; Fig. S4D), after which the two dimers split into two independently diffusing ParB dimers (Fig. S4A-C). This intriguing behavior occurred in about $12 \%$ of the observed ParB molecules that loaded onto the parS site (Fig. 1H). We refer to this type of loading behavior as "ParB-ParB recruitment". We can distinguish ParB-ParB recruitment from simple subsequent loading of ParB proteins at the parS site by evaluating the position along DNA where events

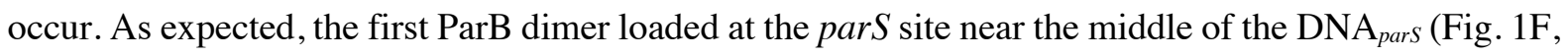
$1 \mathrm{G}$-left), but the loading of the second, recruited ParB dimer occurred later at a position that was, on average, on $5.2 \pm 3.8 \mathrm{~kb}$ (average $\pm \mathrm{SD}, \mathrm{n}=46$ ) away from the $\operatorname{par} S$ site (Fig. 1F, 1G -right). Accordingly, the first ParB loaded at parS site and already diffused over a significant distance before recruiting a new ParB dimer at a distant location (Fig. 1G-right). Interestingly, after ParB-ParB recruitment, we observed that both ParB dimers resided on the DNA for 72 s, i.e., for a similar amount of time as single diffusing ParB before recruitment ( $66 \mathrm{~s}$, Fig. S4C). The recruitment thus approximately doubled the residence time of the initially loaded ParB dimer to $\sim 138$ s (Fig. S4C). Notably, the ParB-ParB recruitment process was highly dependent on the presence of CTP, and occurred hardly at all in the presence of the slowly hydrolysable nucleotide variant CTP $\gamma \mathrm{S}$ (Fig. 1H)(25). Hereafter, we refer to these recruitment events where the second ParB loaded at a DNA position adjacent to the first one, as 'in-cis' (Fig. 2A-left).

By contrast, we observed that ParB-ParB recruitment events can also happen 'in-trans', where the second ParB got loaded onto a DNA position that is genomically distant, but transiently proximal in 3D space via DNA looping (Fig. 2A-right). Since these types of in-trans events could potentially allow for the passage of DNA-bound roadblocks in the crowded in vivo environment, we designed an experiment to test for in-trans recruitment in the presence of DNA-bound roadblocks. We placed a firmly bound 
dCas9 protein on one side of the parS sequence on the DNA (Fig. 2B) and tuned the end-to-end distance of the DNA to $65 \%$ of its total contour length (corresponding to a stretching force of $0.20 \mathrm{pN}$ within the DNA (31)). In these conditions, we observed that the dCas9 roadblock efficiently prevented the diffusion of ParB proteins (Fig. 2C, D and Fig. S5A-E), in line with findings with other DNA-bound roadblocks $(4,7,17)$. However, when repeating the same experiment for a lower end-to-end distance (27\% of the $\mathrm{DNA}_{\text {pars }}$ contour length; $F=0.05 \mathrm{pN}(31)$ ), we observed events where ParB proteins would cross the roadblock and continue one-dimensional diffusion along the DNA on the other side of dCas 9 (Fig. 2E, F and S6A-E, Movie S1). We attribute this behavior to in-trans ParB-ParB recruitment. The bypassing of the Cas9 roadblock was also visibly apparent in the cumulated ParB fluorescence intensity signal, that rapidly increased on the parS side, while the non-parS side displayed discrete increases in

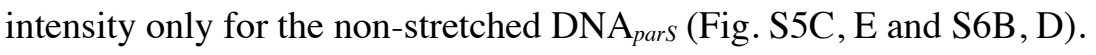

The tension within the DNA appeared to have a significant effect on the success rate of crossing the roadblock. As Fig. 2G displays, the fraction of DNA molecules where ParB successfully passed the dCas9 roadblock decreased from $\sim 80 \%$ at $0.05 \mathrm{pN}$ ( $27 \%$ of the total DNA contour length) to zero at $0.35 \mathrm{pN}$ (75\% contour length (31)). These data are consistent with in-trans ParB-ParB recruitment, where at low stretching forces, regions that are distant in DNA sequence can come into physical contact through bending and looping of the DNA via thermal fluctuations. We further corroborated these findings by observing the ParB-mediated in-trans loading of a new ParB dimer onto a different DNA molecule that was spatially nearby, where this DNA did not contain an endogenous parS site (Fig. $2 \mathrm{H}$, S7). Here, as previously, we bound the $\mathrm{DNA}_{\text {pars }}$ to the surface but then subsequently bound a new DNA

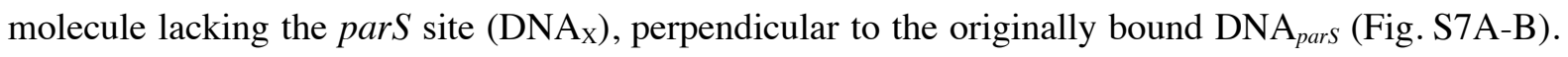

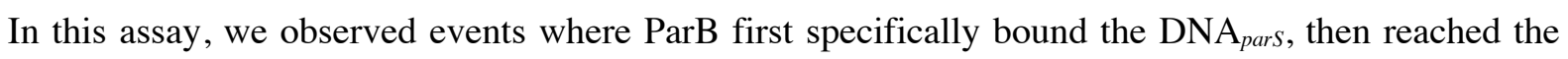
crossing point by random diffusion, where it recruited a new ParB molecule onto the DNAx (Fig. S7C, dashed line), yielding a transfer and subsequent independent one-dimensional diffusion of the newly recruited ParB dimer on the DNAx molecule (Fig. 2H, S7C).

Using coarse-grained molecular dynamics simulations, we modeled the passage of DNA-bound roadblocks by in-trans binding, and found a similar strong force dependence of the passing fraction of roadblocks. We simulated DNA tethers containing a blocking particle which strictly prohibited ParB diffusion through it (Fig. 3A), whereas concurrently ParB could exhibit in-cis or in-trans recruitment with an inbuilt rate (Fig. 3B, see Methods). While in the absence of a roadblock, both in-cis and in-trans recruitment increased the lateral spreading of ParB on the DNA (Fig. S8A), the presence of a roadblock allowed ParB to spread beyond it only through in-trans recruitment (Fig. 3C-D, S8B-D). Upon quantifying the passing fractions at different DNA end-to-end lengths and cis/trans recruitment ratios, we obtained the closest comparison with the experimental data at cis/trans ratio of $\sim 0.1$ (Fig. 2G, 3E). The modeling at very low forces verified the notion that contacts between regions on opposing sides of 
the roadblock occur sufficiently often to allow for ParB to overcome roadblocks via ParB-ParB recruitment.

We found CTP hydrolysis to be crucial for the occurrence of ParB-ParB recruitment (Fig. 1H, S9, Movie S2). To examine this further, we replaced CTP by the poorly hydrolysable CTP $\gamma$ S (Fig. S9A), which allows for the initial loading (clamp closing) but reduces opening of the ParB clamp upon hydrolysis (Fig. S9B-E) $(4,7,19,25)$. This resulted in a low and length-independent passing fraction, indicating an essential role of CTP hydrolysis in the in-trans ParB-ParB recruitment (Fig. S9F-H). As CTP hydrolysis is tightly connected with ParB clamp re-opening and dissociation from the DNA, it is likely that the N-terminal domain of ParB plays a role in the recruitment process. Interestingly, ParB mutants defective in CTP hydrolysis previously showed an altered distribution around par $S$ in $B$. subtilis, with a reduced spreading against the orientation of highly expressed rRNA genes and the DNA replication machinery, and an increased spreading in the opposite direction (19). We suggest that this distribution for the mutant ParB arises from one-dimensional diffusion only (equivalent to the clamping and spreading model), while the spreading of the wild-type protein additionally relies on ParB-ParB recruitment that can overcome head-on encounters with protein machineries such as RNA polymerases or replication forks. Similar observations were made for ParB in C. crescentus (25), whereas by contrast, Myxococcus ParB mutants showed extensive spreading in both directions(32), possibly by unhindered 1D diffusion due to the differences in life cycles (i.e., lower gene transcription and replication rates - see 33,34$)$.

Based on our findings, we propose a model for ParB spreading (Fig. 4) that expands the well-known clamping and sliding model. First, ParB loads onto the parS site and spreads away by one-dimensional diffusion (Fig. 4i), until it hydrolyzes the bound CTP. In our model, however, unlike commonly assumed, the hydrolysis does not necessarily imply the dissociation of ParB from the DNA. Instead, we hypothesize that the ParB dimer may briefly reside in an intermediary state after hydrolyzing CTP, where one of the monomers in not fully engaged in N-terminal dimerization (19). Given abundant CTP in the surrounding buffer, the CDP can now exchange to a new CTP molecule, which leaves the CTPbound free N-terminal part of the protein with two possibilities: (1) either re-connect with the Nterminus on the adjacent monomer of the ParB to re-close the clamp and continue diffusion (Fig. 1E, 4ii) (19), or (2) connect to the N-terminus of a different ParB dimer that is nearby in the solution. The second scenario can lead to recruitment of a second ParB dimer to the nearby DNA, which can occur either in-cis or in-trans (Fig. 4iii). In-trans recruitment, in this fashion, can consequently result in DNAroadblock passing and free diffusion on the other side. The precise molecular mechanism of how the recruited ParB releases the dimer-dimer connection, resulting in separate independent diffusion of both ParB dimers, remains unknown (Fig. 4iii and 4iv). 
Overall, in-trans ParB-ParB recruitment is consistent with the extensive spreading of ParB along genomic DNA that contains many roadblock proteins, Furthermore, it offers an alternative mechanism to the 'stochastic binding' that was proposed in previous models (28) to explain the presence of ParB at large distances from parS sites in in vivo ChIP-seq data. We speculate that ideal hotspots for ParB in-trans recruitment events may additionally be found in regions that are brought into close proximity of a parS site by the action of an SMC complex $(35,36)$. Indeed, recent reports on Hi-C maps detected in strains that were constructed for testing SMC collisions in vivo, showed the co-alignment of DNA flanking a parS-free region which is characteristically only found at $\operatorname{par} S$ sites. Physical contact of DNA with a genomically-distant parS-containing region may lead to in-trans ParB-ParB recruitment and create parS-free ParB-DNA structures suitable for further off-site SMC recruitment (37).

Our findings uncover a new pathway for ParB proteins to cooperatively cover large distances on DNA that is loaded with DNA-binding proteins. It involves the combination of lateral 1D diffusion along the DNA and a new type of CTP-hydrolysis-dependent ParB loading (ParB-ParB recruitment) which can occur irrespective of the parS site. Both our experimental and simulation data showed that in-trans ParB-ParB recruitment can account for overcoming DNA-bound roadblocks at low forces where DNA forms a fluctuating polymer blob that facilitates frequent DNA-DNA encounters. Since both the ParB concentration and the DNA-contact frequency are higher in the tightly packed genome within a bacterial cell compared to our single-molecule experiments, we expect ParB-ParB recruitment to be a common mechanism in vivo, where it may facilitate the collective spreading distance of ParB proteins on the protein-bound DNA. 


\section{References:}

1. J. Livny, Y. Yamaichi, M. K. Waldor, Distribution of centromere-like parS sites in bacteria: Insights from comparative genomics. J. Bacteriol. 189 (2007), doi:10.1128/JB.01239-07.

2. G. J. Bean, S. T. Flickinger, W. M. Westler, M. E. McCully, D. Sept, D. B. Weibel, K. J. Amann, A22 disrupts the bacterial actin cytoskeleton by directly binding and inducing a lowaffinity state in MreB. Biochemistry (2009), doi:10.1021/bi900014d.

3. D. C. H. Lin, A. D. Grossman, Identification and characterization of a bacterial chromosome partitioning site. Cell (1998), doi:10.1016/S0092-8674(00)81135-6.

4. Y. M. Soh, I. F. Davidson, S. Zamuner, J. Basquin, F. P. Bock, M. Taschner, J. W. Veening, P. de Los Rios, J. M. Peters, S. Gruber, Self-organization of parS centromeres by the ParB CTP hydrolase. Science. 366, 1129-1133 (2019).

5. M. Osorio-Valeriano, F. Altegoer, W. Steinchen, S. Urban, Y. Liu, G. Bange, M. Thanbichler, ParB-type DNA Segregation Proteins Are CTP-Dependent Molecular Switches. Cell (2019), doi:10.1016/j.cell.2019.11.015.

6. I. V. Surovtsev, C. Jacobs-Wagner, Subcellular Organization: A Critical Feature of Bacterial Cell Replication. Cell. 172 (2018), , doi:10.1016/j.cell.2018.01.014.

7. F. de A. Balaguer, C. Aicart-Ramos, G. L. M. Fisher, S. de Bragança, E. M. Martin-Cuevas, C. L. Pastrana, M. S. Dillingham, F. Moreno-Herrero, CTP promotes efficient ParB-dependent DNA condensation by facilitating one-dimensional diffusion from parS. Elife. 10, e67554 (2021).

8. B. W. Chen, M. H. Lin, C. H. Chu, C. E. Hsu, Y. J. Sun, Insights into ParB spreading from the complex structure of Spo0J and parS. Proc. Natl. Acad. Sci. U. S. A. 112 (2015), doi:10.1073/pnas.1421927112.

9. A. Sanchez, D. I. Cattoni, J. C. Walter, J. Rech, A. Parmeggiani, M. Nollmann, J. Y. Bouet, Stochastic Self-Assembly of ParB Proteins Builds the Bacterial DNA Segregation Apparatus. Cell Syst. 1 (2015), doi:10.1016/j.cels.2015.07.013.

10. D. Song, K. Rodrigues, T. G. W. Graham, J. J. Loparo, A network of cis and trans interactions is required for ParB spreading. Nucleic Acids Res. 45 (2017), doi:10.1093/nar/gkx271.

11. B. E. Funnell, ParB partition proteins: Complex formation and spreading at bacterial and plasmid centromeres. Front. Mol. Biosci. (2016), , doi:10.3389/fmolb.2016.00044.

12. B. Guilhas, J.-C. Walter, J. Rech, G. David, N. Walliser, J. Palmeri, C. Mathieu-Demaziere, A. Parmeggiani, J.-Y. Bouet, A. Le Gall, M. Nollmann, ATP-Driven Separation of Liquid Phase Condensates in Bacteria. SSRN Electron. J. (2019), doi:10.2139/ssrn.3462750.

13. T. G. W. Graham, X. Wang, D. Song, C. M. Etson, A. M. van Oijen, D. Z. Rudner, J. J. Loparo, ParB spreading requires DNA bridging. Genes Dev. 28 (2014), doi:10.1101/gad.242206.114.

14. A. G. Vecchiarelli, K. C. Neuman, K. Mizuuchi, A propagating ATPase gradient drives transport of surface-confined cellular cargo. Proc. Natl. Acad. Sci. U. S. A. 111 (2014), doi:10.1073/pnas.1401025111.

15. H. C. Lim, I. V. Surovtsev, B. G. Beltran, F. Huang, J. Bewersdorf, C. Jacobs-Wagner, Evidence for a DNA-relay mechanism in ParABS-mediated chromosome segregation. Elife (2014), doi:10.7554/eLife.02758.

16. A. S. B. Jalal, T. B. K. Le, Bacterial chromosome segregation by the ParABS system. Open Biol. 10 (2020), doi:10.1098/rsob.200097.

17. A. S. Jalal, N. T. Tran, T. B. Le, ParB spreading on DNA requires cytidine triphosphate in vitro. Elife. 9 (2020), doi:10.7554/eLife.53515.

18. A. M. Breier, A. D. Grossman, Whole-genome analysis of the chromosome partitioning and sporulation protein Spo0J (ParB) reveals spreading and origin-distal sites on the Bacillus subtilis chromosome. Mol. Microbiol. 64 (2007), doi:10.1111/j.1365-2958.2007.05690.x.

19. H. Antar, Y.-M. Soh, S. Zamuer, F. P. Bock, A. Anchimiuk, P. De Los Rios, S. Gruber, Relief of ParB autoinhibition by parS DNA catalysis and ParB recycling by CTP hydrolysis promote bacterial centromere assembly. Sci. Adv. (2021), doi:10.1101/2021.05.05.442573.

20. V. Lagage, F. Boccard, I. Vallet-Gely, Regional Control of Chromosome Segregation in 
Pseudomonas aeruginosa. PLoS Genet. 12 (2016), doi:10.1371/journal.pgen.1006428.

21. K. Böhm, G. Giacomelli, A. Schmidt, A. Imhof, R. Koszul, M. Marbouty, M. Bramkamp, Chromosome organization by a conserved condensin-ParB system in the actinobacterium Corynebacterium glutamicum. Nat. Commun. 11 (2020), doi:10.1038/s41467-020-15238-4.

22. J. H. Baek, S. V. Rajagopala, D. K. Chattoraj, Chromosome segregation proteins of vibrio cholerae as transcription regulators. MBio. 5 (2014), doi:10.1128/mBio.01061-14.

23. O. Rodionov, M. ŁObocka, M. Yarmolinsky, Silencing of genes flanking the P1 plasmid centromere. Science. 283 (1999), doi:10.1126/science.283.5401.546.

24. H. Murray, H. Ferreira, J. Errington, The bacterial chromosome segregation protein Spo0J spreads along DNA from parS nucleation sites. Mol. Microbiol. 61 (2006), doi:10.1111/j.13652958.2006.05316.x.

25. A. S. Jalal, N. T. Tran, C. E. Stevenson, A. Chimthanawala, A. Badrinarayanan, D. M. Lawson, T. B. Le, A CTP-dependent gating mechanism enables ParB spreading on DNA. Elife. 10 (2021), doi:10.7554/elife.69676.

26. R. L. Ohniwa, Y. Ushijima, S. Saito, K. Morikawa, Proteomic analyses of nucleoid-associated proteins in escherichia coli, pseudomonas aeruginosa, bacillus subtilis, and staphylococcus aureus. PLoS One. 6 (2011), doi:10.1371/journal.pone.0019172.

27. F. Kunst, N. Ogasawara, I. Moszer, A. M. Albertini, G. Alloni, V. Azevedo, M. G. Bertero, P. Bessières, A. Bolotin, S. Borchert, R. Borriss, L. Boursier, A. Brans, M. Braun, S. C. Brignell, S. Bron, S. Brouillet, C. V. Bruschi, B. Caldwell, V. Capuano, N. M. Carter, S. K. Choi, J. J. Codani, I. F. Connerton, N. J. Cummings, R. A. Daniel, F. Denizot, K. M. Devine, A. Düsterhöft, S. D. Ehrlich, P. T. Emmerson, K. D. Entian, J. Errington, C. Fabret, E. Ferrari, D. Foulger, C. Fritz, M. Fujita, Y. Fujita, S. Fuma, A. Galizzi, N. Galleron, S. Y. Ghim, P. Glaser, A. Goffeau, E. J. Golightly, G. Grandi, G. Guiseppi, B. J. Guy, K. Haga, J. Haiech, C. R. Harwood, A. Hénaut, H. Hilbert, S. Holsappel, S. Hosono, M. F. Hullo, M. Itaya, L. Jones, B. Joris, D. Karamata, Y. Kasahara, M. Klaerr-Blanchard, C. Klein, Y. Kobayashi, P. Koetter, G. Koningstein, S. Krogh, M. Kumano, K. Kurita, A. Lapidus, S. Lardinois, J. Lauber, V. Lazarevic, S. M. Lee, A. Levine, H. Liu, S. Masuda, C. Mauël, C. Médigue, N. Medina, R. P. Mellado, M. Mizuno, D. Moestl, S. Nakai, M. Noback, D. Noone, M. O’Reilly, K. Ogawa, A. Ogiwara, B. Oudega, S. H. Park, V. Parro, T. M. Pohl, D. Portetelle, S. Porwollik, A. M. Prescott, E. Presecan, P. Pujic, B. Purnelle, G. Rapoport, M. Rey, S. Reynolds, M. Rieger, C. Rivolta, E. Rocha, B. Roche, M. Rose, Y. Sadaie, T. Sato, E. Scanlan, S. Schleich, R. Schroeter, F. Scoffone, J. Sekiguchi, A. Sekowska, S. J. Seror, P. Serror, B. S. Shin, B. Soldo, A. Sorokin, E. Tacconi, T. Takagi, H. Takahashi, K. Takemaru, M. Takeuchi, A. Tamakoshi, T. Tanaka, P. Terpstra, A. Tognoni, V. Tosato, S. Uchiyama, M. Vandenbol, F. Vannier, A. Vassarotti, A. Viari, R. Wambutt, E. Wedler, H. Wedler, T. Weitzenegger, P. Winters, A. Wipat, H. Yamamoto, K. Yamane, K. Yasumoto, K. Yata, K. Yoshida, H. F. Yoshikawa, E. Zumstein, H. Yoshikawa, A. Danchin, The complete genome sequence of the gram-positive bacterium Bacillus subtilis. Nature. 390 (1997), doi:10.1038/36786.

28. J. C. Walter, J. Rech, N. O. Walliser, J. Dorignac, F. Geniet, J. Palmeri, A. Parmeggiani, J. Y. Bouet, Physical Modeling of a Sliding Clamp Mechanism for the Spreading of ParB at Short Genomic Distance from Bacterial Centromere Sites. iScience. 23 (2020), doi:10.1016/j.isci.2020.101861.

29. R. E. Debaugny, A. Sanchez, J. Rech, D. Labourdette, J. Dorignac, F. Geniet, J. Palmeri, A. Parmeggiani, F. Boudsocq, V. Anton Leberre, J. Walter, J. Bouet, A conserved mechanism drives partition complex assembly on bacterial chromosomes and plasmids. Mol. Syst. Biol. 14 (2018), doi:10.15252/msb.20188516.

30. M. Ganji, I. A. Shaltiel, S. Bisht, E. Kim, A. Kalichava, C. H. Haering, C. Dekker, Real-time imaging of DNA loop extrusion by condensin. Science. 360, 102-105 (2018).

31. C. Bouchiat, M. D. Wang, J. F. Allemand, T. Strick, S. M. Block, V. Croquette, Estimating the persistence length of a worm-like chain molecule from force-extension measurements. Biophys. J. 76 (1999), doi:10.1016/S0006-3495(99)77207-3.

32. M. Osorio-Valeriano, F. Altegoer, C. K. Das, W. Steinchen, G. Panis, L. Connolley, G. Giacomelli, H. Feddersen, L. Corrales-Guerrero, P. Giammarinaro, J. Hanßmann, M. 
Bramkamp, P. H. Viollier, S. Murray, L. V Schäfer, G. Bange, M. Thanbichler, The CTPase activity of ParB acts as a timing mechanism to control the dynamics and function of prokaryotic DNA partition complexes. Mol. Cell. 81, 3992-4007.e10 (2021).

33. J. Muñoz-Dorado, A. Moraleda-Muñoz, F. J. Marcos-Torres, F. J. Contreras-Moreno, A. B. Martin-Cuadrado, J. M. Schrader, P. I. Higgs, J. Pérez, Transcriptome dynamics of the myxococcus xanthus multicellular developmental program. Elife. 8 (2019), doi:10.7554/eLife.50374.

34. S. Thiery, C. Kaimer, The Predation Strategy of Myxococcus xanthus. Front. Microbiol. 11 (2020), , doi:10.3389/fmicb.2020.00002.

35. X. Wang, T. B. K. Le, B. R. Lajoie, J. Dekker, M. T. Laub, D. Z. Rudner, Condensin promotes the juxtaposition of dna flanking its loading site in Bacillus subtilis. Genes Dev. 29, 16611675 (2015).

36. X. Wang, H. B. Brandão, T. B. K. Le, M. T. Laub, D. Z. Rudner, Bacillus subtilis SMC complexes juxtapose chromosome arms as they travel from origin to terminus. Science. (2017), doi:10.1126/science.aai8982.

37. H. B. Brandão, Z. Ren, X. Karaboja, L. A. Mirny, X. Wang, DNA-loop-extruding SMC complexes can traverse one another in vivo. Nat. Struct. Mol. Biol. 28 (2021), doi:10.1038/s41594-021-00626-1.

\section{References only in supplementary meterial:}

38. B. Pradhan, R. Barth, E. Kim, I. F. Davidson, B. Bauer, T. van Laar, W. Yang, J.-K. Ryu, J. van der Torre, J.-M. Peters, C. Dekker, SMC complexes can traverse physical roadblocks bigger than their ring size. bioRxiv (2021).

39. E. Kim, J. Kerssemakers, I. A. Shaltiel, C. H. Haering, C. Dekker, DNA-loop extruding condensin complexes can traverse one another. Nature (2020), doi:10.1038/s41586-020-20675 .

40. J. Schreiber, pomegranate: Fast and flexible probabilistic modeling in python. J. Mach. Learn. Res. 18 (2018). 
Acknowledgments: We thank Alejandro Martin Gonzalez, Eugene Kim, Florian Patrick Bock and Richard Janissen for useful discussions, and Richard Janissen for sharing software for force estimation on the DNA tether based on the end-to-end distance.

Funding: We acknowledge funding support by the European Research Council Advanced Grant 883684 as well as the Netherlands Organisation for Scientific Research (NWO/OCW), as part of the NanoFront and BaSyC programs. DM is a Royal Society University Research Fellow and is supported by the European Research Council Starting Grant on Topologically Active Polymers (Ref. 947918).

Author contributions: Conceptualization: MT, SG, CD. Experiments: MT. Formal analysis: MT, BP, RB. Methodology: MT, JvdT, CD, MP, DM. Protein purification: HA, YMS. Software: BP. Simulations: MP, DM. Visualization: MT. Funding acquisition: DM, SG, CD. Supervision: DM, SG, CD. Writing - original draft: MT, CD. Writing - review \& editing: MT, HA, YMS, MP, RB, BP, JvdT, $\mathrm{DM}, \mathrm{SG}, \mathrm{CD}$.

Competing interests: Authors declare that they have no competing interests.

Data and materials availability: All data are available in the main text or the supplementary material. 
A

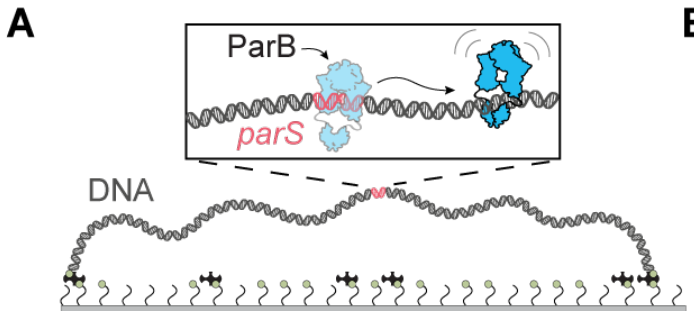

C
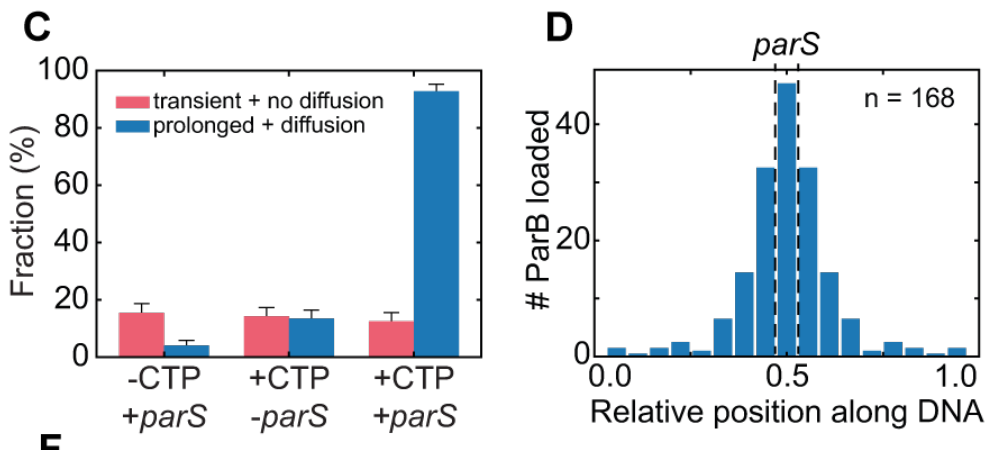

$\mathbf{F}$

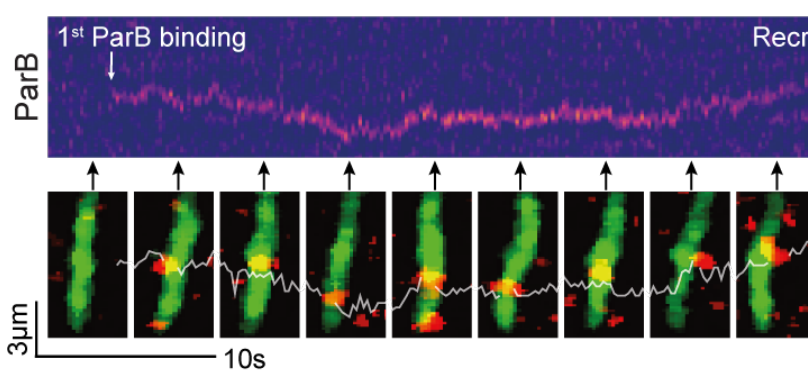

G

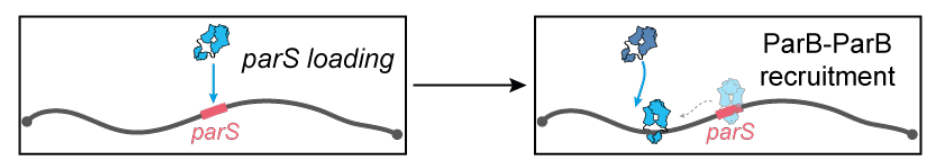

Distance $[\mathrm{kbp}]$

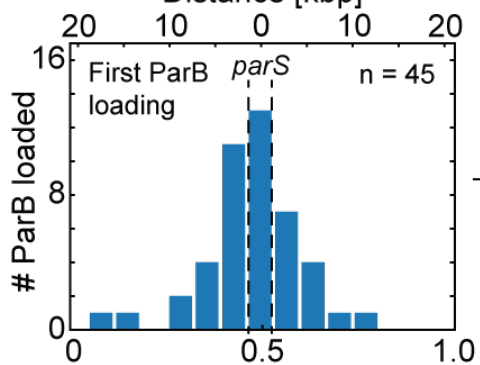

Rel. position along DNA

Distance $[\mathrm{kbp}]$

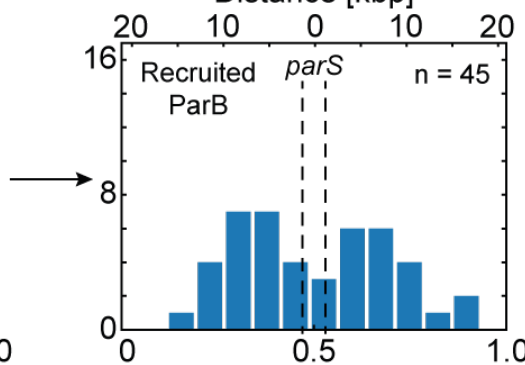

Rel. position along DNA

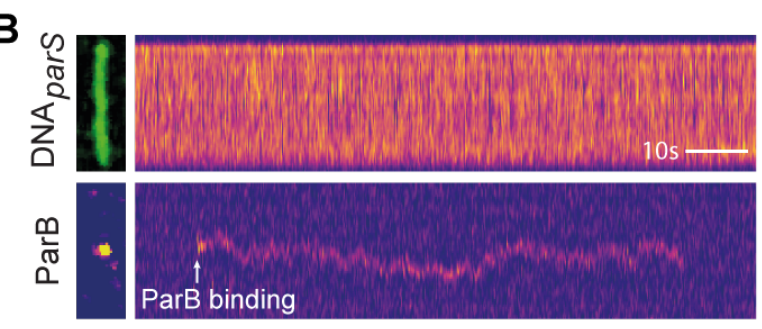

E

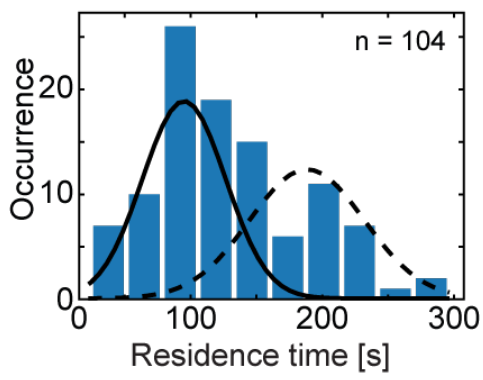

$\mathbf{H}$

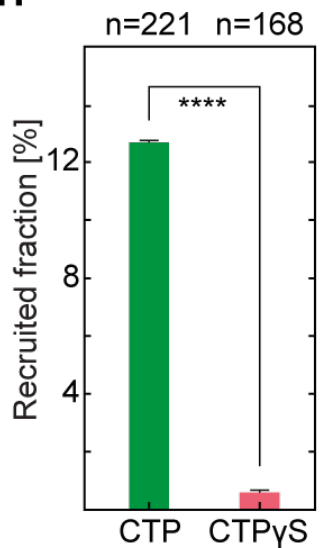

Figure 1. DNA-bound ParB dimers can recruit new ParB dimers independently of parS.

A) Schematic representation of DNA pars $_{\text {s }}$ that is tethered at both its ends to a surface, see also Fig. S1.

B) Kymographs for DNA pars $_{\text {stained with SytoxGreen (top) and ParB }}{ }^{\text {alexa647 }}$ (bottom). Left images show single-frame snapshots of the DNA and ParB at the moment of binding. White arrow indicates ParB loading. C) Fraction of DNA tethers with transient binding with no diffusion and the fraction showing prolonged binding with diffusion of ParB molecules, for various conditions $(n=123,133,112$ from left to right). Error bars represent binomial proportion confidence intervals. D) Histogram showing 
bioRxiv preprint doi: https://doi.org/10.1101/2021.11.02.466941; this version posted November $2,2021$. The copyright holder for this preprint (which was not certified by peer review) is the author/funder, who has granted bioRxiv a license to display the preprint in perpetuity. It is made available under aCC-BY-NC-ND 4.0 International license.

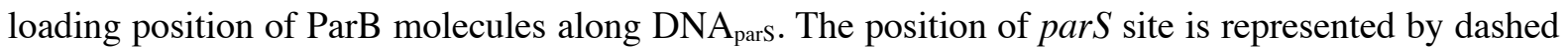
lines, $\mathrm{n}=168$. E) Residence times of diffusing ParB molecules after binding DNA $\mathrm{Dars}_{\mathrm{S}}(\mathrm{n}=127)$. Gaussian fits are represented in black. F) Top: kymograph for ParB ${ }^{\text {alexa647 }}$. White arrows indicate first ParB-dimer binding, second ParB-dimer binding, and splitting. Bottom: Snapshot images, taken at time points indicated by black arrows. White line indicates the single-particle-tracking trace. G) Histograms of ParB loading positions. Left: First ParB loading at parS site. Right: loading position of the recruited second ParB dimer. Top panels are illustrative schemes. H) Recruitment rate in presence of CTP (green) or CTP $\gamma \mathrm{S}$ (red). Error bars represent binominal confidence intervals. Statistical significance calculated using chi-squared test for a binomial distribution, $\chi^{2}(n=389)=20.196, p<0.00001$. 
A
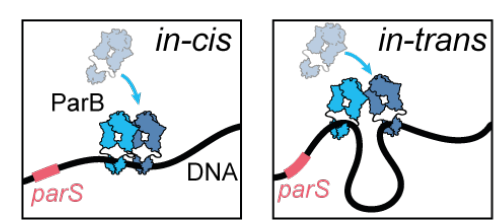

C
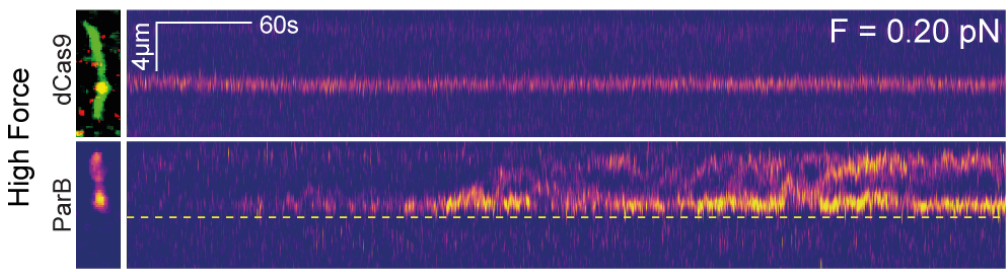

E
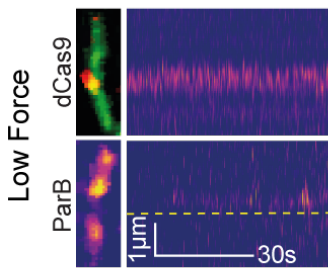

G

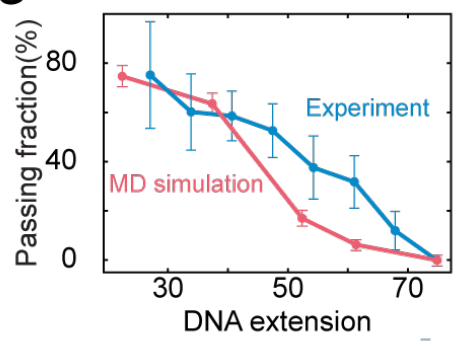

[\% of countour length]

$\mathbf{H}$

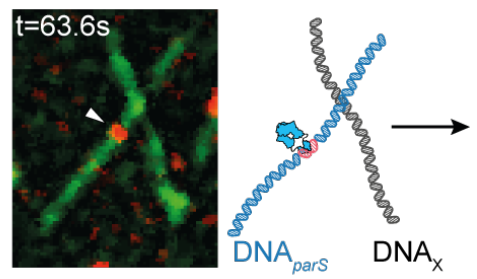

B

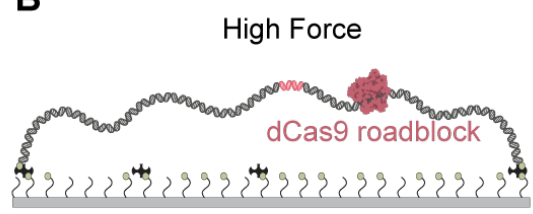

D

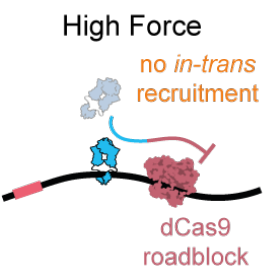

$\mathbf{F}$
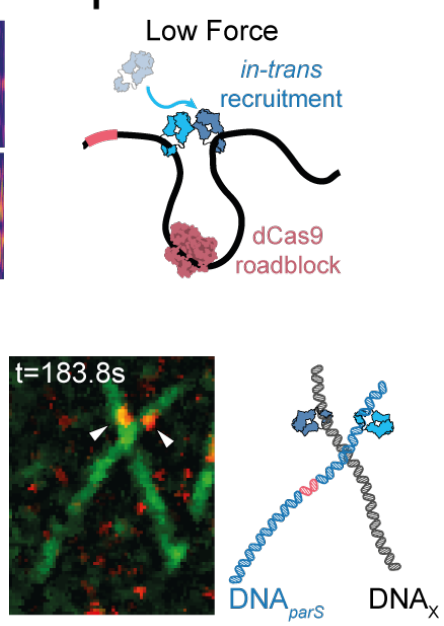

Figure 2. ParB can bypass roadblocks by in-trans ParB-ParB recruitment.

A) Illustration of in-cis (left) and in-trans (right) recruitment events. B) Schematic of the roadblock experiment for a large (left, high force) and small $\mathrm{DNA}_{\text {pars }}$ end-to-end distance (right, low force). C) Kymograph for dCas9 $9^{\text {alexa549 }}$ (top), and ParB ${ }^{\text {alexa647 }}$ (bottom) for DNA $\mathrm{Dars}_{\text {at }} 0.20 \mathrm{pN}$. Snapshots of dCas $9^{\text {alexa549/SytoxGren }} \mathrm{DNA}_{\text {pars }}$ overlay and ParB ${ }^{\text {alexa647 }}$ signal are provided on the left. Yellow dashed line indicates the position of the $\mathrm{dCas}^{\text {alexa549 }}$. D) Cartoon of the $\mathrm{DNA}_{\text {pars }}$ at high force where ParB molecules cannot be recruited across the roadblock. E) Same as panel C but data at a force of $0.05 \mathrm{pN}$. White arrow denotes a passing event. F) Same as panel D but for low force where ParB molecules can be recruited in-trans across the dCas9-roadblock. G) Fraction of DNA molecules that exhibit ParB dimers passing the dCas9-roadblock, displayed versus stretching length of the DNA. Error bars represent binomial proportion confidence intervals (blue, $\mathrm{n}=122$ ), and standard deviation from simulation cycles (pink, n=128). H) Left: Snapshot of the $\mathrm{DNA}_{\text {pars }}-\mathrm{DNA}_{\mathrm{x}}$ crossing at the time of the first ParB binding to parS site, $\mathrm{t}=105 \mathrm{~s}$. Right: Snapshot of the same field of view at time $\mathrm{t}=306 \mathrm{~s}$, where a second ParB was recruited onto the $\mathrm{DNA}_{\mathrm{x}}$ by in trans recruitment. White arrows indicate the positions of ParB dimers. Cartoon representations are provided on the right of the snapshot frames. 
A

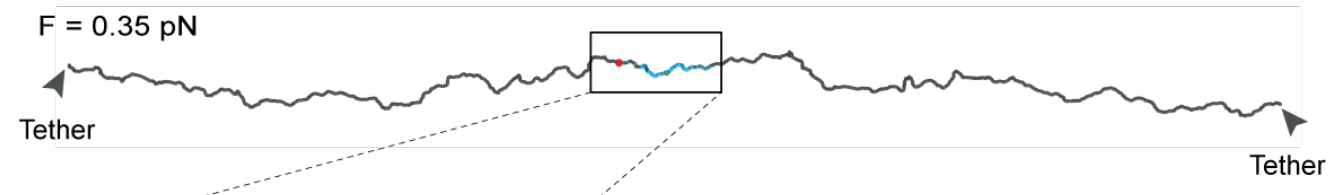

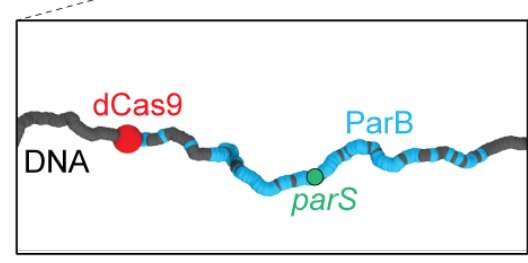

(i)

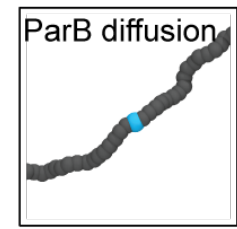

C

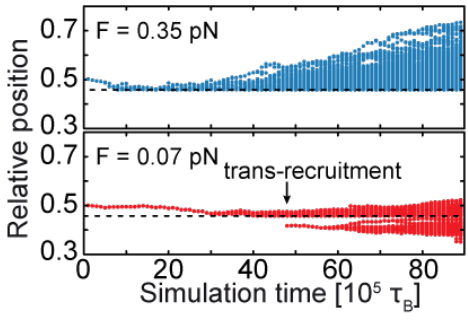

\section{B}

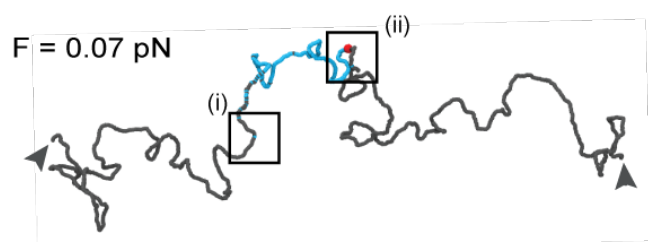

(ii)
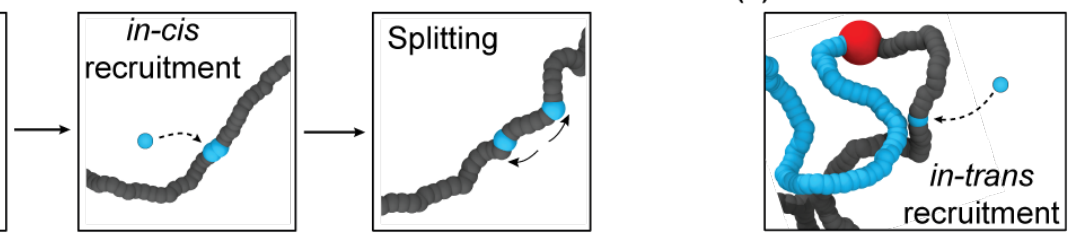

E
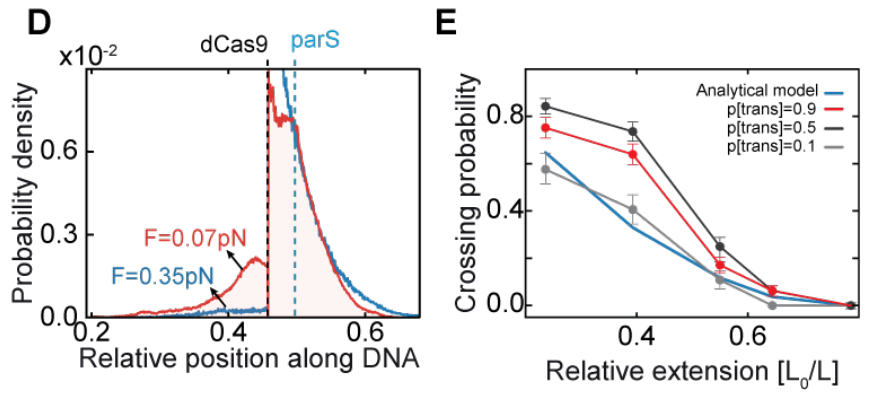

Figure 3. DNA looping via thermal fluctuations allows in-trans ParB-ParB recruitment and spreading across roadblocks.

A) Single frame snapshot of the DNA tethers in the MD simulations. DNA tether end-to-end length $11 \mu \mathrm{m}$ (i.e., $75 \%$ contour length, force of $0.35 \mathrm{pN}$ ). Zoomed region highlights the region proximal to parS site. Tether has a parS site in the middle (green), and simulated dCas9-roadblock on one side (orange). ParB represented in yellow. B) Single frame snapshot of low-force DNA molecule in MD simulations. DNA tether end-to-end length $5.5 \mu \mathrm{m}$ (i.e., $35 \%$ contour length, force of $0.07 \mathrm{pN}$ ). Highlighted regions (a) and (b) show in-cis and in-trans recruitment events, respectively. Color-coding same as in panel A. C) Simulated kymographs representing distributions of ParB positions during the simulation. Top: kymograph for DNA tether at $0.35 \mathrm{pN}$; bottom: same at $\mathrm{F}=0.07 \mathrm{pN}$. Black arrow indicates in-trans recruitment event. D) Histogram of cumulated ParB probability density from MD simulations at different tether lengths (blue $11 \mu \mathrm{m}$, red $5.5 \mu \mathrm{m}$ ) averaged over $\mathrm{n}=64$ simulations. Dashed line indicates the position of dCas9 roadblock. E) Fraction of simulated DNA tethers that exhibit ParB dimers passing the dCas9-roadblock at different cis/trans ratios. The theoretical passing rate (integration of polymer looping probability, see Fig. S8E) is shown in blue. Error bars represent standard deviation from simulation cycles $(\mathrm{n}=128)$. 


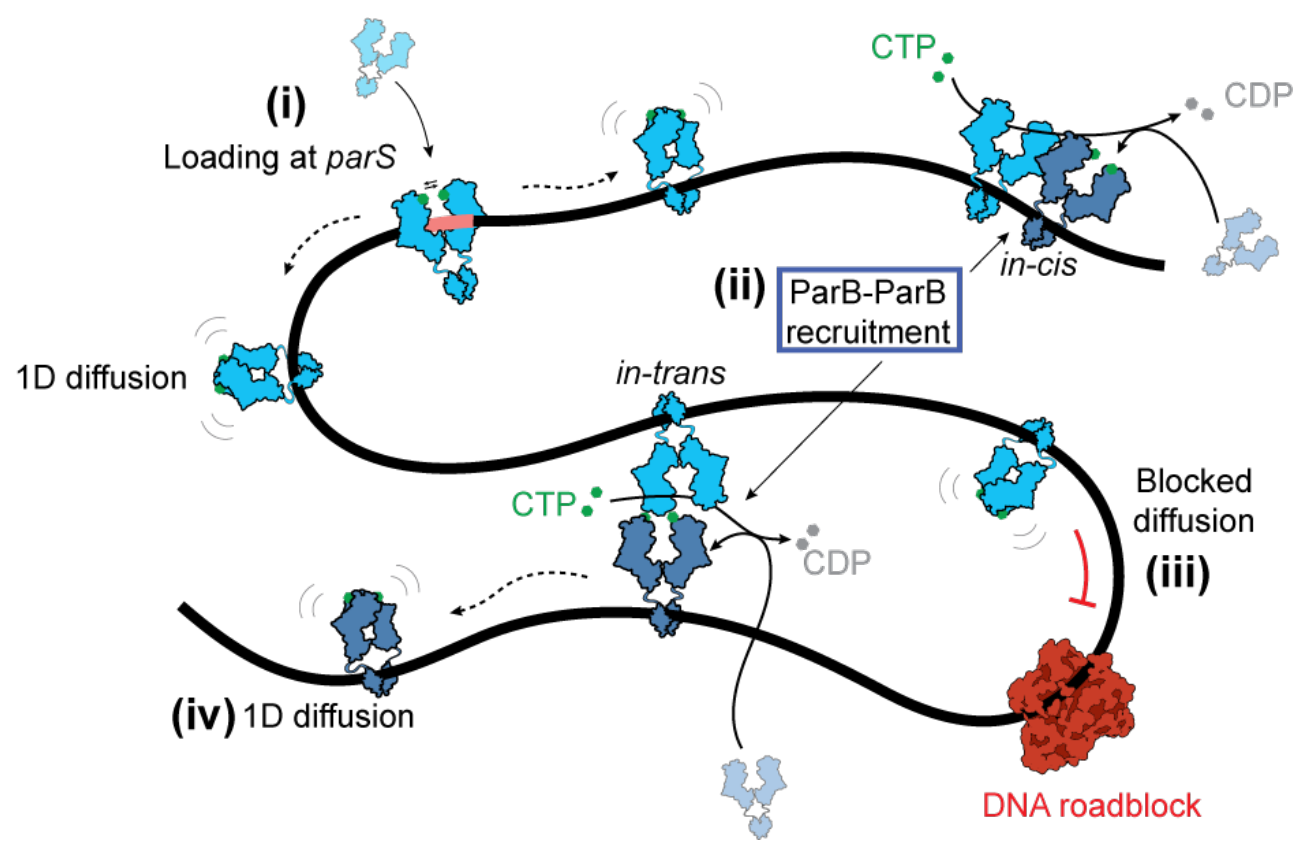

Figure 4. Model of ParB spreading and recruitment.

Cartoon of the various ParB modalities on DNA: (i) ParB dimer loads at the parS site, dimerizes upon closing its N-termini, losing its affinity to the parS-site, and diffusing away as a closed clamp. Subsequently (ii), ParB hydrolyzes CTP and enters an intermediary state where it can bind and recruit another ParB dimer to a nearby DNA, which can occur in-cis or in-trans. By exchanging CDP and $\mathrm{P}_{\mathrm{i}}$ after hydrolysis for a new CTP molecule from solution, ParB dimer can also re-form a clamped structure and continue diffusing. While one-dimensional diffusion is blocked by DNA-roadblocks (iii), ParB dimers that are recruited in-trans across the roadblock can continue diffusion along the DNA, yielding an expanded spreading of ParB along DNA. 